\title{
PKconverter: $\mathrm{R}$ package to convert the pharmacokinetic parameters
}

\author{
Hyeseon Cho and Eun-Kyung Lee \\ Department of Statistics, Ewha Womans University, Seoul 03760, Korea \\ ${ }^{*}$ Correspondence: E. K. Lee; Tel: +82-2-3277-6857, Fax: +82-2-3277-3607, E-mail: lee.eunk@ewha.ac.kr
}

Check for updates

Received 25 Feb 2019

Revised 19 Mar 2019

Accepted 2 Apr 2019

\section{Keywords}

Pharmacokinetic model, Pharmacokinetic parameter, Population analysis,

$\mathrm{R}$,

Shiny

pISSN: 2289-0882

elSSN: 2383-5427
Population pharmacokinetic analysis and modeling procedures typically require estimates of both population and individual pharmacokinetic parameters. However, only some of these parameters are contained in models and only parameters in the model can be estimated. In this paper, we introduce a new R package, PKconverter, to calculate pharmacokinetic parameters using the relationships among them. After fitting the model, other parameters can be calculated from the functional relationship among the parameters. PKconverter provides the functions to calculate whole parameters along with a Shiny application for converting the parameters. With this package, it is also possible to calculate the standard errors of the other parameters that are not in the model and estimate individual parameters simultaneously.

\section{Introduction}

Pharmacokinetics $(\mathrm{PK})$ is the study of drug absorption, distribution, metabolism, and excretion. PK models explain how drug concentration changes as a drug moves through the different compartments of the body. An understanding of basic PK parameters is essential for PK modeling and analysis. All parameters are considered, but only some are used by models. Those not used can be calculated from the parameters in the model using the functional relationships among the PK parameters.

After fitting the PK model, the estimated parameters can be converted to other parameters using their functional relations. Most modelers use the "Convert.xls" file provided by Shafer (1999) at www.nonmemcourse.com to convert pharmacokinetic parameters in one-, two-, or three-compartment models. The Microsoft Excel file consists of five different calculation sheets, each of which has different input and output parameters. A user can choose the sheet according to the known input parameters and the number of compartments in the model and then input estimated parameters. The other parameters are returned by the spreadsheet.

Copyright (C) 2019 Translational and Clinical Pharmacology

(a) It is identical to the Creative Commons Attribution Non-Commercial License (http://creativecommons.org/licenses/by-nc/3.0/).

@ This paper meets the requirement of KS X ISO 9706, ISO 9706-1994 and ANSI/NISO Z.39.48-1992 (Permanence of Paper).

Reviewer

This article was reviewed by the peer experts who are not TCP editors.
Several difficulties are associated with this tool. First, Microsoft Excel is commercial software. Second, while the converted parameters are in the spreadsheet and the numbers can be checked, it is not easy to use these numbers for further analysis without typing them into another file. Third, even though it provides converted parameters in a spreadsheet for a given population parameter, it is sometimes necessary to know individual PK parameters. With this Excel file, we can convert only one set of parameters for one individual at a time. To convert a set of parameters for all individuals, the estimated parameters for one individual must be entered, the results checked, and the procedure repeated for each individual. Fourth, this Excel file does not provide the approximate standard error of converted parameters.

To address these limitations, we developed a new $\mathrm{R}$ package, PKconverter. This package provides the user with the converted parameters and the approximated standard error of the converted parameters using the delta method. It also converts individual parameters with the standard errors simultaneously and can save the entire result as a single file. With the Shiny [1] graphical user interface (GUI), a novice R user can easily calculate the converted parameters. PKconverter (https://cran. r-project.org/web/packages/PKconverter/index.html) is on $\mathrm{R}$ repository CRAN and everyone can download freely and use it. We also put this on the web (https://ek-lee.shinyapps.io/PKconverter/) that the user can use on the web without install $\mathrm{R}$ or PKconverter packages. 
Here we first introduce the pharmacokinetic models and parameters, and the relationships among the pharmacokinetic parameters in each compartment model. We then explain the delta method and how to use it to calculate the approximated standard errors of the converted parameters. Finally, we describe how to convert the parameters with the several types of input parameters. We also introduce the Shiny application interface for PKconverter.

\section{Methods}

PK studies the time course of drug concentration in different body spaces and the relationship between concentration and the time course of drug action.[2-7] Drug absorption, distribution, metabolism, and elimination are fundamental aspects of pharmacokinetic modeling and analysis. The pharmacokinetic model explains the transfer of a drug from its absorption site to the body and the various steps involved in the distribution and elimination of the drug in the body. It is essential to check and interpret all pharmacokinetic parameters.

The volume of distribution (V) defines the apparent space or volume that a drug distributes. Clearance (CL) defines the volume of blood that is completely cleared of drug content per unit time. It describes the capacity of irreversible elimination of a compound from plasma and is a consequence of a combination of perfusion, diffusion, filtration, metabolism, and transport processes of the clearing organ. The elimination rate constant $(\mathrm{K})$ relates to the amount of drug in the body that is eliminated per unit of time. The half-life of the drug $(\mathrm{t} 1 / 2)$ is the time required to reduce by half the amount of a drug remaining in the body. A fractional coefficient is defined as a true coefficient multiplied by the volume of distribution. The sum of all fractional coefficients is always 1 ; for example, for a three-compartment model, F.A + F.B + F.C $=1$. The exponents $\alpha, \beta$, and $\gamma$ represent the slopes of the decay in the plot of drug concentration vs. time. The half-lives are calculated by $\log (2)$ divided by the corresponding exponents.

Although all PK parameters should be considered for analysis, only some of these parameters are used in the model, and the others can be calculated by the functional relations with them. Therefore, after fitting the pharmacokinetic model, the other parameters must be calculated with these estimated parameters, which are not used in the model.

\section{Compartment model}

In pharmacometrics, the compartmental model is categorized by the number of compartments needed to describe a drug's absorption, distribution, metabolism, and excretion in the body.[8] There are one-, two-, and three-compartment models. These models are used to predict the time course of drug concentrations in the body. In a one-compartment model, $C_{\mathrm{t}}$ is the drug concentration at time $t, C_{0}$ is the initial concentration, and $K_{10}$ is the elimination rate.

In the pharmacometrics area, a basic type of model is the compartmental model that is categorized by the number of compartments needed to describe the drug's absorption, distribution, metabolism, and excretion in the body.[8] There are one-compartmental, two-compartmental, and multi-compartmental models. We usually use up to three-compartmental model. These models are used to predict the time course of drug concentrations in the body. The one-compartmental model is

$$
\begin{aligned}
C_{t} & =C_{0} e^{-k_{10} t} \\
& =A e^{-\alpha t} .
\end{aligned}
$$

(2) is the general form of the one-compartment model. A is the true coefficient, and $\alpha$ is the exponent. These two parameters have functional relation with various pharmacokinetic parameters V1, CL1, $\mathrm{K}_{10}, \mathrm{t}_{1 / 2, \alpha}$, etc.

$$
\begin{gathered}
V 1=\frac{1}{A}, \quad C L 1=\frac{\alpha}{A}, \quad K_{10}=\alpha, \\
t_{1 / 2, \alpha}=\frac{\log 2}{\alpha}, \quad V d_{s S}=V 1=\frac{1}{A}, F . A=A \times V 1=1 .
\end{gathered}
$$

For example, a one-compartment intravenous bolus model can be represented in three ways:

$$
\begin{aligned}
C_{t} & =\frac{\text { Dose }}{V 1} e^{-K_{10} t} \\
& =\frac{\text { Dose } \cdot K_{10}}{C L 1} e^{-K_{10} t} \\
& =\frac{\text { Dose }}{V 1} e^{-\frac{C L 1}{V 1} t},
\end{aligned}
$$

where CL1 is the clearance, V1 is the volume of distribution, and $K_{10}$ is the elimination rate constant. Because the pharmacokinetic parameters V1, CL1, and $\mathrm{K}_{10}$ have a functional relation of $\mathrm{CL} 1=\mathrm{V} 1 \cdot \mathrm{K}_{10}$, the model equation can be represented by various form with only two pharmacokinetic parameters, and the other parameters can be found after fitting one of three equations. Figure 1 and Figure 2 provide the plots of the functional relationships among the pharmacokinetic parameters.

The general form of the two-compartmental model is

$$
C_{t}=A e^{-\alpha t}+B e^{-\beta t},
$$

where $A$ or $B$ is used for $C_{0}$ in the one-compartmental model, and $\alpha$ or $\beta$ are used for the elimination rate. With these four parameter estimates, 12 more parameters can be calculated with the following equations:

$$
\begin{aligned}
& K_{21}=\frac{A \alpha+B \beta}{A+B}, \quad K_{10}=\frac{\alpha \beta}{K_{21}}, \quad k_{12}=\alpha+\beta-k_{21}-\frac{\alpha \beta}{k_{21}}, \\
& V 1=\frac{1}{A+B}, \quad V 2=\frac{V 1}{K_{21}}\left(\alpha+\beta-K_{21}-\frac{\alpha \beta}{K_{21}}\right), V d_{s s}=V 1+V 2,
\end{aligned}
$$




$$
\begin{gathered}
C L 1=V 1 \times K_{10}, C L 2=V 2 \times K_{12}, \\
t_{1 / 2, \alpha}=\frac{\log 2}{\alpha}, \quad t_{1 / 2, \beta}=\frac{\log 2}{\beta}, \\
F . A=A \times V 1, \quad F . B=B \times V 1 .
\end{gathered}
$$

Similar relations can be defined in the three-compartment model.

Table 1 summarized important pharmacokinetic parameters in each compartmental model and Table 2 summarized input parameters for each compartmental model in each spreadsheet in Shiner's "Convert.xls" file.

\section{Delta method}

In pharmacometrics, the maximum likelihood estimation (MLE) of a PK parameter can be found with NONMEM or

\section{one comp: V1, $\mathrm{Cl} 1$, and $\mathrm{k} 10$}

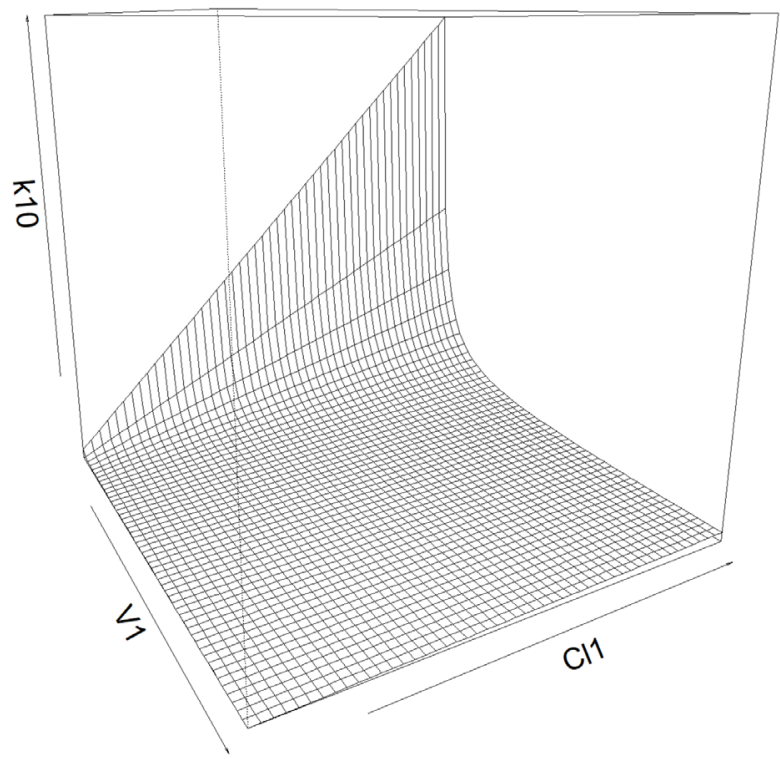

Figure 1. The relationship among PK parameters? V1, Cl1, and k10 in one compartment model. other software. Most software involved in generating estimates of PK parameters is based on maximum likelihood theory. If $\theta$ is the PK parameter and $\hat{\theta}$ is the MLE of $\theta$, then, by the properties of MLE, $[9,10]$

$$
\sqrt{n}(\hat{\theta}-\theta) \sim N(0, \operatorname{Var}(\hat{\theta}))
$$

To calculate the other PK parameters that are the functions of MLEs, let $\mathrm{f}(\theta)$ be the other PK parameters. The estimate of $f(\theta)$ is $\hat{f}(\theta)-f(\hat{\theta})$ and $\operatorname{Var}(f(\hat{\theta}))=f^{\prime}(\hat{\theta})^{2} \operatorname{Var}(\hat{\theta})$, the delta method.[11] If the dimension of $\theta, \mathrm{q}$, is greater than 1 ,

$$
\operatorname{Var}(f(\hat{\theta}))=G(\hat{\theta})^{T} \operatorname{Var}(\hat{\theta}) G(\hat{\theta})
$$

where $G(\hat{\theta})=\frac{\partial f(\theta)}{\partial \theta}$, q-dimensional vector.

\section{one comp: $\mathrm{V}, \mathrm{Cl}$, and $\mathrm{t}$ _alpha}

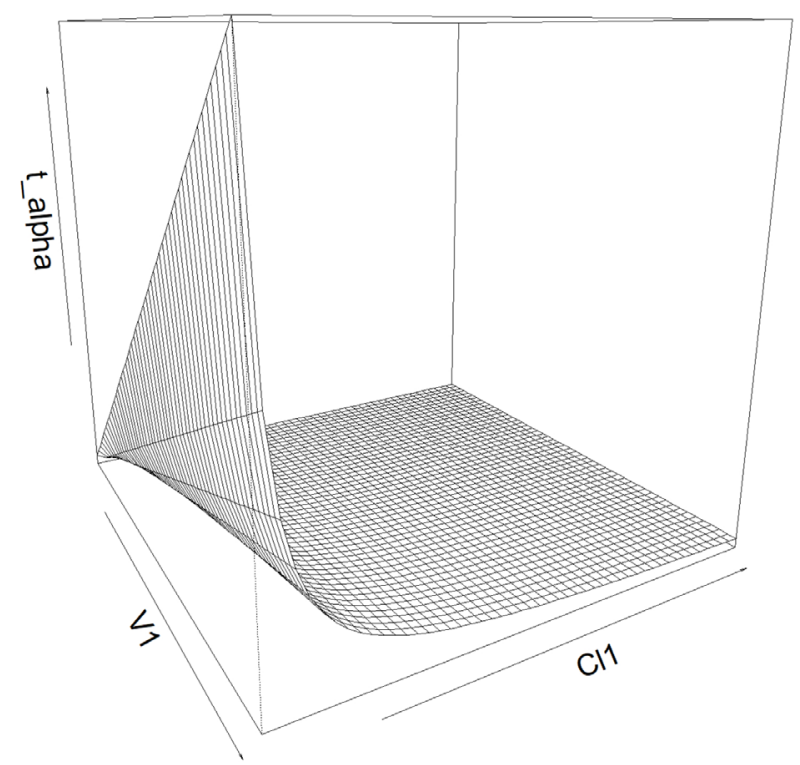

Figure 2. The relationship among PK parameters? V1, Cl1, and t_alpha in one compartment model.

Table 1. Important pharmacokinetic parameters in each compartment model

\begin{tabular}{lccc}
\hline \multicolumn{1}{c}{ Type } & One compartment & Two compartment & Three compartment \\
\hline Volume of distribution & $V 1$ & $V 1, V 2$ & $V 1, V 2, V 3$ \\
Vdss & $V d_{s s}$ & $V d_{s s}$ & $V d_{s s}$ \\
Clearance & $C L 1$ & $C L 1, C L 2$ & $C L 1, C L 2, C L 3$ \\
Rate constant & $K_{10}$ & $K_{10}, K_{12}, K_{21}$ & $K_{10}, K_{12}, K_{21}, K_{13}, K_{31}$ \\
Half-life & $t_{1 / 2, a}$ & $t_{1 / 2, a}, t_{1 / 2, \beta}$ & $t_{1 / 2, a}, t_{1 / 2, \beta}, t_{1 / 2, \gamma}$ \\
True coefficient & $\mathrm{A}$ & $\mathrm{A}, \mathrm{B}$ & $\mathrm{A}, \mathrm{B}, \mathrm{C}$ \\
Fractional coefficient & $\mathrm{F} . \mathrm{A}$ & F.A, F.B & F.A, F.B, F.B \\
Exponent & $\alpha$ & $\alpha, \beta$ & $\alpha, \beta, \gamma$ \\
\hline
\end{tabular}




\section{Population parameter convert}

We provide five different models with known parameters for three kinds of compartment models. In the following subsection, we summarize the input and output parameters of each model. We then provide functions to calculate PK parameters with the approximated standard errors and supply a Shiny application for each model. Table 2 lists input parameters and $\mathrm{R}$ function names for each model.

Model 1 uses the volume of distributions and the clearances as the input parameters. For one-compartmental model, with the known parameters $V 1$ and $C L 1$, the other parameters can be calculated with the following equations:

$$
\begin{gathered}
V d_{s s}=V 1, \quad A=\frac{1}{V 1}, \quad \alpha=\frac{C l 1}{V 1}, \\
t_{1 / 2, \alpha}=\frac{\log 2}{k_{10}}=\log 2 \cdot \frac{V 1}{C l 1}, \quad k_{10}=\frac{C l 1}{V 1}, F . A=A \cdot V 1
\end{gathered}
$$

For the two-compartmental model, with the know parameters $V 1, V 2, C L 1$, and $C L 2$, we can calculate the others:

$$
\begin{gathered}
V d_{S S}=V 1+V 2, k_{10}=\frac{c l 1}{V 1}, k_{12}=\frac{c l 2}{V 1}, k_{21}=\frac{c l 2}{V 2} . \\
a_{0}=k_{10} k_{21}, a_{1}=-\left(k_{10}+k_{12}+k_{21}\right), \\
\alpha=\frac{-a_{1}+\sqrt{a_{1}{ }^{2}-4 a_{0}}}{2}, \beta=\frac{-a_{1}-\sqrt{a_{1}{ }^{2}-4 a_{0}}}{2}, \\
A=\frac{k_{21}-\alpha}{\beta-\alpha} \times \frac{1}{V 1}, B=\frac{k_{21}-\beta}{\alpha-\beta} \times \frac{1}{V 1}, \quad t_{1 / 2, \alpha}=\frac{\log 2}{\alpha}, \quad t_{1 / 2, \beta}=\frac{\log 2}{\beta},
\end{gathered}
$$

For the three-compartmental model, the following equations can be used:

$$
\begin{gathered}
V d_{s s}=V 1+V 2+V 3, \\
k_{10}=\frac{C l 1}{V 1}, \quad k_{12}=\frac{C l 2}{V 1}, \quad k_{13}=\frac{C l 3}{V 1}, \quad k_{21}=\frac{C l 2}{V 2}, \quad k_{31}=\frac{C l 3}{V 3}, \\
A=\frac{\left(k_{21}-\alpha\right)\left(k_{31}-\alpha\right)}{V 1(\alpha-\beta)(\alpha-\gamma)}, \quad B=\frac{\left(k_{21}-\beta\right)\left(k_{31}-\beta\right)}{V 1(\beta-\alpha)(\beta-\gamma)}, \quad C=\frac{\left(k_{21}-\gamma\right)\left(k_{31}-\gamma\right)}{V 1(\gamma-\alpha)(\gamma-\beta)}, \\
t_{1 / 2, \alpha}=\frac{\log 2}{\alpha}, \quad t_{1 / 2, \beta}=\frac{\log 2}{\beta}, \quad t_{1 / 2, \gamma}=\frac{\log 2}{\gamma} .
\end{gathered}
$$

where $\alpha, \beta, \gamma$ are determined by size order of root $_{1}, \operatorname{root}_{2}$, root $_{3}$ from the largest to smallest.

\begin{tabular}{|c|c|c|c|}
\hline Model & Compartment & Input & Function name \\
\hline \multirow{3}{*}{1} & 1 & $V 1, C L 1$ & OneComp_Volume_Clearance \\
\hline & 2 & $V_{1}, V 2, C L 1, C L 2$ & TwoComp_Volume_Clearance \\
\hline & 3 & $V 1, V 2, V 3, C L 1, C L 2, C L 3$ & ThreeComp_Volume_Clearance \\
\hline \multirow{3}{*}{2} & 1 & $V 1, K_{10}$ & OneComp_Volume_RateConstant \\
\hline & 2 & $V 1, K_{10}, K_{12}, K_{21}$ & TwoComp_Volume_RateConstant \\
\hline & 3 & $V 1, K_{10}, K_{12}, K_{21}, K_{13}, K_{31}$ & ThreeComp_Volume_RateConstant \\
\hline \multirow{3}{*}{3} & 1 & $C L 1, t_{1 / 2, a}$ & OneComp_Volume_Clearance_HalfLife \\
\hline & 2 & $V 1, C L 1, t_{1 / 2, a}, t_{1 / 2, \beta}$ & TwoComp_Volume_Clearance_HalfLife \\
\hline & 3 & $V 1, C L 1, t_{12, a}, t_{12, \beta}, t_{1 / 2, \gamma}, V_{s s}$ & ThreeComp_Volume_Clearance_HalfLife \\
\hline \multirow{3}{*}{4} & 1 & $\mathrm{~A}, \alpha$ & OneComp_Coefficient_Exponent \\
\hline & 2 & $\mathrm{~A}, \mathrm{~B}, \alpha, \beta$ & TwoComp_Coefficient_Exponent \\
\hline & 3 & $\mathrm{~A}, \mathrm{~B}, \mathrm{C}, \alpha, \beta, \gamma$ & ThreeComp_Coefficient_Exponent \\
\hline \multirow{3}{*}{5} & 1 & $V 1, a$ & OneComp_Volume_Exponent \\
\hline & 2 & $V 1, K_{21}, \alpha, \beta$ & TwoComp_Volume_Exponent \\
\hline & 3 & $V 1, K_{21}, K_{31}, \alpha, \beta, \gamma$ & ThreeComp_Volume_Exponent \\
\hline
\end{tabular}

$$
\begin{gathered}
\operatorname{root}_{1}=-r_{2} \cos (p h i)+\frac{a_{2}}{3}, \text { root }_{2}=-r_{2} \cos \left(\text { phi }+\frac{2 \pi}{3}\right)+\frac{a_{2}}{3}, \text { root }_{3}=-r_{2} \cos \left(\text { phi }+\frac{4 \pi}{3}\right)+\frac{a_{2}}{3}, \\
a_{0}=k_{10} k_{21} k_{31}, \quad a_{1}=k_{10} k_{31}+k_{21} k_{31}+k_{21} k_{13}+k_{10} k_{21}+k_{31} k_{12}, \\
a_{2}=k_{10}+k_{12}+k_{13}+k_{21}+k_{31}, \\
p=a_{1}-\frac{a_{2}{ }^{2}}{3}, \quad q=\frac{2 a_{2}{ }^{3}}{27}-\frac{a_{1} a_{2}}{3}+a_{0}, \\
r_{1}=\sqrt{-\frac{p^{3}}{27}}, p h i=\frac{1}{3} \operatorname{acos}\left(-\frac{q}{2 r_{1}}\right), \quad r_{2}=2 \cdot \exp \left(\frac{\log \left(r_{1}\right)}{3}\right) .
\end{gathered}
$$

Model 2 uses the volume of distribution of 1st compartment and rate constants, model 3 uses the volume of distribution and the clearance of the 1st compartment and half-lives, model 4 uses the coefficients and exponents, and model 5 uses the volume of distribution of the $1^{\text {st }}$ compartment, exponents, and some of the rate constants. The detailed formula to calculate the other parameters are in.[12]

Table 2. Summary of input parameters and function names in each compartment in each model 


\section{Results}

For each model and each compartment, we provide an R function. The function names for each model in each compartment are supplied in Table 2. For one-compartmental model with Model 1, OneComp_Volume_Clearance function is used as follows:

OneComp_Volume_Clearance $(\mathrm{V} 1=8, \mathrm{Cl} 1=4, \mathrm{~V} 1 . \mathrm{sd}=0.01, \mathrm{Cl} 1 . \mathrm{sd}=0.01)$

\#\#

\#\# Vdss

\#\# V1

\#\# C11

\#\# k10

\#\# alpha

\#\# t_alpha

\#\# True_A

\#\# Frac_A
Parameter Estimate Std.err

Vdss $8.000000 \quad 0.010000000$

V1 $8.000000 \quad 0.010000000$

Cl1 $4.000000 \quad 0.010000000$

k10 $0.500000 \quad 0.001397542$

alpha $0.500000 \quad 0.001397542$

True_A $0.125000 \quad 0.001562500$

Frac_A 1.0000000 .000000000 t_alpha $1.386294 \quad 0.003874811$
For the two-compartment model with Model 1, TwoComp_Volume_Clearance $(V 1=5, V 2=50, C l 1=3.5, C 12=2.5$,
V1. $\mathrm{sd}=0.01, \mathrm{~V} 2 . \mathrm{sd}=0.1, \mathrm{Cl1} . \mathrm{sd}=0.01, \mathrm{Cl} 2 . \mathrm{sd}=0.01)$

$\begin{array}{lrrr}\text { \#\# } & \text { Parameter } & \text { Estimate } & \text { Std.err } \\ \text { \#\# Vdss } & \text { Vdss } & 55.000000000 & 1.004988 \mathrm{e}-01 \\ \text { \#\# V1 } & \text { V1 } & 5.000000000 & 1.000000 \mathrm{e}-02 \\ \text { \#\# V2 } & \text { V2 } & 50.000000000 & 1.000000 \mathrm{e}-01 \\ \text { \#\# C11 } & \text { Cl1 } & 3.500000000 & 1.000000 \mathrm{e}-02 \\ \text { \#\# C12 } & \text { C12 } & 2.500000000 & 1.000000 \mathrm{e}-02 \\ \text { \#\# k10 } & \text { k10 } & 0.700000000 & 2.441311 \mathrm{e}-03 \\ \text { \#\# k12 } & \text { k12 } & 0.500000000 & 2.236068 \mathrm{e}-03 \\ \text { \#\# k21 } & \text { k21 } & 0.050000000 & 2.236068 \mathrm{e}-04 \\ \text { \#\# alpha } & \text { alpha } & 1.221343022 & 3.765020 \mathrm{e}-03 \\ \text { \#\# beta } & \text { beta } & 0.028656978 & 9.280865 \mathrm{e}-05 \\ \text { \#\# t_alpha } & \text { t_alpha } & 0.567528670 & 1.749514 \mathrm{e}-03 \\ \text { \#\# t_beta } & \text { t_beta } & 24.187727779 & 7.833451 \mathrm{e}-02 \\ \text { \#\# True_A } & \text { True_A } & 0.196421016 & 4.007910 \mathrm{e}-04 \\ \text { \#\# True_B } & \text { True_B } & 0.003578984 & 2.157918 \mathrm{e}-05 \\ \text { \#\# Frac_A } & \text { Frac_A } & 0.982105079 & 1.140066 \mathrm{e}-04 \\ \text { \#\# Frac_B } & \text { Frac_B } & 0.017894921 & 1.140066 \mathrm{e}-04\end{array}$

\section{$\begin{array}{lllllll}\text { PK Parameter Converter } & \text { Model } 1 & \text { Model } 2 & \text { Model } 3 & \text { Model } 4 & \text { Model } 5 & \text { Indiv. Parameter Converter }\end{array}$}

\section{Select your model}

MODEL TYPE :
- One compartment model
- Two compartment model
- Three compartment model

\section{Enter your estimate and std.err}

$$
\text { Estimate / Std.err Covariance }
$$

V1 Estimate
8

\section{Cl1 Estimate}

4
V1 Std.err

\begin{tabular}{|l} 
V1 Std.err \\
0.01
\end{tabular}

Cl1 Std.err

\subsection{1}

\section{Model 1: Volumes and Clearances}

\section{One compartment model}

\begin{tabular}{llrr} 
& Parameter & Estimate & Std.err \\
\hline Volume & Vdss & 8.0000 & 0.0100 \\
& V1 & 8.0000 & 0.0100 \\
\hline Clearnace & Cl1 & 4.0000 & 0.0100 \\
\hline Micro Rate Constant & k10 & 0.5000 & 0.0014 \\
\hline Exponent & alpha & 0.5000 & 0.0014 \\
\hline Half-lives & t_alpha & 1.3863 & 0.0039 \\
\hline True Coeffficient & True_A & 0.1250 & 0.0016 \\
\hline Fractional Coefficient & Frac_A & 1.0000 & 0.0000
\end{tabular}

Save results as a file

File type:

- Excel (CSV)

Text (tab separated)

Text (Space Separated)

$\downarrow$ Save results to file

Figure 3. Main GUI of Shiny application for Pharmacokinetic Parameter Converter - Model 1. 


\section{Web Interface}

We provide a Shiny application[1] to accompany the R package. Figure 3 shows the main GUI of a Shiny application for a pharmacokinetic parameter converter with a Model 1 tab. There are five tabs in this application, and each model consists of one model. The user can choose a one, two, or three-compartment model from the "MODEL TYPE" radio button. After entering the values of the volume of distributions and clearances, the gray-shaded output area is automatically changed as it calculates the other parameters. PKconverter then calculates the standard errors of each parameter using the delta method. In the "Covariance" tab, the covariance between the volume of distribution and the clearance can be entered and considered in the calculation of the standard errors of the other parameters.

\section{Individual parameter converter}

Figure 4 is the GUI for converting individual parameters simultaneously. After selecting the compartment model and data type, users can upload an individual pharmacokinetic parameter file. After matching the name of input PK parameters with the user's input file, the other parameters are automatically calculated and presented. The user can save these results as a file.

\section{Discussion}

In this paper, we introduce a new $\mathrm{R}$ package, PKconverter, to calculate pharmacokinetic parameters using the relationships among them. After fitting the model, other parameters can be calculated from the functional relationship among the parameters. PKconverter provides the functions to calculate whole

\section{PK Parameter Converter $\quad$ Model $1 \quad$ Model 2 Model $3 \quad$ Model $4 \quad$ Model 5 Indiv. Parameter Converter}

\section{MODEL TYPE : \\ - One compartment model \\ Two compartment model \\ Three compartment model}

Select your compartment model

\section{Select your input data type \\ DATA TYPE : \\ - Model 1: Volumes and Clearances \\ Model 2: V1, Rate Constants \\ Model 3: V1, Vdss, Cl, half-lives \\ Model 4: Coefficients and Exponents \\ Model 5: V1, Exponents,K21,K31}

\section{Open your individual PK parameters}

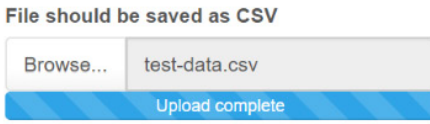

\section{Match PK parameters}

ID

ID $\quad-$

v1

V1 -

Cl1

$\mathrm{CL} \quad-$

t Save individual parameters to file

\begin{tabular}{rrrrrrrrr} 
ID & Vdss & V1 & Cl1 & k10 & alpha & t_alpha & True_A & Frac_A \\
\hline 1.00 & 0.16 & 0.16 & 0.03 & 0.17 & 0.17 & 4.16 & 6.19 & 1.00 \\
\hline 2.00 & 0.21 & 0.21 & 0.03 & 0.12 & 0.12 & 5.79 & 4.66 & 1.00 \\
\hline 3.00 & 0.28 & 0.28 & 0.04 & 0.14 & 0.14 & 4.98 & 3.56 & 1.00 \\
\hline 4.00 & 0.37 & 0.37 & 0.04 & 0.10 & 0.10 & 6.68 & 2.72 & 1.00 \\
\hline 5.00 & 0.29 & 0.29 & 0.04 & 0.15 & 0.15 & 4.62 & 3.43 & 1.00 \\
\hline 6.00 & 0.23 & 0.23 & 0.03 & 0.14 & 0.14 & 4.82 & 4.42 & 1.00 \\
\hline 7.00 & 0.37 & 0.37 & 0.05 & 0.13 & 0.13 & 5.40 & 2.74 & 1.00 \\
\hline 8.00 & 0.30 & 0.30 & 0.04 & 0.14 & 0.14 & 4.96 & 3.33 & 1.00 \\
\hline 9.00 & 0.26 & 0.26 & 0.03 & 0.13 & 0.13 & 5.21 & 3.81 & 1.00 \\
\hline 10.00 & 0.20 & 0.20 & 0.03 & 0.14 & 0.14 & 4.98 & 4.95 & 1.00 \\
\hline 11.00 & 0.05 & 0.05 & 0.02 & 0.32 & 0.32 & 2.18 & 20.91 & 1.00 \\
\hline 12.00 & 0.04 & 0.04 & 0.01 & 0.34 & 0.34 & 2.06 & 24.37 & 1.00 \\
\hline 13.00 & 0.10 & 0.10 & 0.02 & 0.25 & 0.25 & 2.82 & 10.10 & 1.00 \\
\hline 14.00 & 0.10 & 0.10 & 0.02 & 0.22 & 0.22 & 3.13 & 10.24 & 1.00 \\
\hline 15.00 & 0.05 & 0.05 & 0.02 & 0.36 & 0.36 & 1.91 & 20.98 & 1.00 \\
\hline 16.00 & 0.15 & 0.15 & 0.03 & 0.19 & 0.19 & 3.74 & 6.65 & 1.00 \\
\hline 17.00 & 0.14 & 0.14 & 0.02 & 0.18 & 0.18 & 3.88 & 7.28 & 1.00 \\
\hline 18.00 & 0.14 & 0.14 & 0.03 & 0.22 & 0.22 & 3.20 & 7.04 & 1.00 \\
\hline 19.00 & 0.15 & 0.15 & 0.03 & 0.19 & 0.19 & 3.67 & 6.67 & 1.00 \\
\hline 20.00 & 0.18 & 0.18 & 0.03 & 0.15 & 0.15 & 4.69 & 5.41 & 1.00 \\
\hline 21.00 & 0.16 & 0.16 & 0.03 & 0.16 & 0.16 & 4.33 & 6.38 & 1.00 \\
\hline 22.00 & 0.15 & 0.15 & 0.02 & 0.13 & 0.13 & 5.20 & 6.65 & 1.00 \\
\hline 23.00 & 0.22 & 0.22 & 0.03 & 0.14 & 0.14 & 5.06 & 4.52 & 1.00 \\
\hline 24.00 & 0.07 & 0.07 & 0.01 & 0.20 & 0.20 & 3.49 & 13.83 & 1.00 \\
\hline 25.00 & 0.20 & 0.20 & 0.02 & 0.09 & 0.09 & 7.46 & 4.96 & 1.00 \\
\hline 26.00 & 0.12 & 0.12 & 0.02 & 0.15 & 0.15 & 4.54 & 8.06 & 1.00 \\
\hline 27.00 & 0.13 & 0.13 & 0.02 & 0.13 & 0.13 & 5.26 & 7.68 & 1.00 \\
\hline & & & & & & & & \\
\hline 1.00 &
\end{tabular}

Figure 4. Main GUI of Shiny application for Pharmacokinetic Parameter Converter - Individual parameter converter. 
parameters along with a Shiny application for converting the parameters. With this package, it is also possible to calculate the standard errors of the other parameters that are not in the model and estimate individual parameters simultaneously. It can be convenient not only for calculating population parameters but also for calculating individual parameters. To check the reliability of the calculations in this package, we compare the result of the converted parameters with the pmxTools package. The pmxTools package provides the functions to convert the parameters. However, these functions do not provide the standard errors of converted pharmacokinetic parameters. To confirm the reliability of the calculation of the converted parameters, we use Theoph data and nlme functions in the nlme package. The results are provided as supplementary materials.

This package can read an input file with individual parameters and convert them simultaneously. All results in the Shiny application can be saved as an output file. With this saved file, we can easily use the calculated parameters for further analysis. PKconverter (https://cran.r-project.org/web/packages/PKconverter/index.html) is on R repository CRAN and everyone can download freely and use it. Shiny app is also on the web (https:// ek-lee.shinyapps.io/PKconverter/) that the user can use on the web without install $\mathrm{R}$ or PKconverter packages. We hope that this package can help pharmacometricians analyze and understand the compartmental models.

\section{Acknowledgments}

This research was supported by Basic Science Research Program through the National Research Foundation of Korea (NRF) funded by the Ministry of Education (2015R1D1A1A01056790).

\section{Conflict of interest}

- Authors: Nothing to declare

- Reviewers: Nothing to declare

- Editors: Nothing to declare

\section{References}

1. Chang W, Cheng J, Allaire JJ, Xie Y, McPherson J. Shiny: web application framework for R. R package version $0.11,2015$

2. DiPiro JT. Concepts in clinical pharmacokinetics. ASHP, 2010

3. Rowland M, Tozer TN, Derendorf H, Hochhaus G. Clinical pharmacokinetics and pharmacodynamics: concepts and applications. Wolters Kluwer Health/Lippincott William \& Wilkins Philadelphia, PA, 2011

4. Gabrielsson J, Weiner D. Pharmacokinetic and pharmacodynamic data analysis: concepts and applications, volume 2. CRC Press, 2001.

5. Benet LZ, Zia-Amirhosseini P. Basic principles of pharmacokinetics. Toxicol Pathol 1995;23: 115-123. doi: 10.1177/019262339502300203.

6. Mould D, Upton R. Basic concepts in population modeling, simulation, and model-based drug development. CPT Pharmacometrics Syst Pharmacol 2012;1:1-14. doi: 10.1038/psp

7. Mould D, Upton RN. Basic concepts in population modeling, simulation, and model-based drug development-part 2: introduction to pharmacokinetic modeling methods. CPT Pharmacometrics Syst Pharmacol 2013;2:1-14. doi: 10.1038/psp

8. Shargel L, Andrew B, Wu-Pong S. Applied biopharmaceutics \& pharmacokinetics. Appleton \& Lange Stamford, 1999

9. Lehmann EL. Elements of large-sample theory. Springer Science \& Business Media, 2004

10. Taboga T. Lectures on probability theory and mathematical statistics. CreateSpace Independent Publishing Platform, 2012

11. Casella G, Berger RL. Statistical inference, volume 2. Duxbury Pacific Grove, CA, 2002

12. Cho H. PKconverter: shiny application to convert the PK parameters, Dissertation, Ewha Womans University, 2018 